1 DOSSIER THÉMATIQUE 1

SIUE DEUS SIUE DEA. DÉNOMINATIONS DIVINES DANS LES MONDES GREC ET SÉMITIQUE : UNE APPROCHE PAR LE GENRE

DOSSIER THÉMATIQUE 2

TRADITION ET TRANSMISSION DANS L'ANTIQUITÉ : RÉFLEXIONS INTERDISCIPLINAIRES

98 Claire CAMBerlein, Efstathia Dionysopoulou \& Thibault Foulon

Introduction. La tradition et sa transmission : positionnements théoriques

105 Mélissa Leuzy

Consigner ou fabriquer la légende d'Alexandre le Grand ? Arrien face à la tradition dans l'Anabase

117 Héloïse SMETS

Un autre regard sur l'archaïsme dans les sépultures privées de l'Égypte pharaonique

128 Laura WALDVOGEL

Nouveaux indices de variabilité au sein des traditions funéraires de l'ouest du Rubané occidental

141 Grégoire BLANC

Praxis citationnelle et co-construction du discours scientifique dans les Naturales Quaestiones de Sénèque (livres II \& III)

150 Anthony GLAISE

Polémiques, traditions et identités : réflexions autour des Discours contre les juifs et les judaïsants de Jean Chrysostome

159 ACTUALITÉ DE LA RECHERCHE

QUOI DE NEUF À L'OUEST DE STRASBOURG ? KOENIGSHOFFEN : ÉTAT DES LIEUX ET DÉCOUVERTES RÉCENTES

195 VARIA 


\title{
POLÉMIQUES, TRADITIONS ET IDENTITÉS : RÉFLEXIONS AUTOUR DES DISCOURS CONTRE LES JUIFS ET LES JUDAÏSANTS DE JEAN CHRYSOSTOME
}

\author{
Anthony GLAISE \\ Docteur en Lettres classiques \\ Université de Tours \\ UMR 7323 - CESR \\ anthony.glaise@etu.univ-tours.fr
}

RÉSUMÉ

Dans son Histoire de l'antisémitisme, Léon Poliakov qualifie les Discours contre les juifs de Jean Chrysostome (vers 347-407) de « diatribes d'une violence inimaginable » censées illustrer I'« antisémitisme théologique » des Pères de l'Église. Cette analyse prend cependant insuffisamment en compte la tradition, ou plutôt les traditions qui irriguent ces textes du Père antiochien. Ainsi, s'ils sont d'abord imprégnés par la tradition de la polémique antijudaïque, qui marque la littérature chrétienne depuis ses débuts, les textes de Jean Chrysostome se placent aussi dans la tradition de la rhétorique païenne véhiculée dans I'enseignement qu'ont reçu les auteurs chrétiens du IV siècle. Cette tradition littéraire et son exigence de clarté et d'usage raisonné des sentiments ont été pour notre auteur des outils précieux pour faire valoir son point de vue au sein de la cité d'Antioche, alors marquée par une vie religieuse intense et troublée. Reconsidérer et réévaluer ces deux traditions au sein de l'œuvre de Jean Chrysostome, c'est redonner à ces textes leur signification réelle : la contribution d'un prêtre antiochien à la construction d'une identité chrétienne. Replacer les Discours contre les juifs dans le contexte de leur élaboration et dans la chaîne ininterrompue des traditions dont ils sont tributaires permet donc

\section{MOTS-CLÉS}

Jean Chrysostome, polémique antijudaïque, rhétorique antique, christianisme, Antiquité tardive. d'en faire non pas la preuve d'un « antisémitisme théologique », comme le disait Poliakov, mais un témoignage d'une réflexion intense et vivante sur l'identité chrétienne à la fin de l'Antiquité.
In his History of Antisemitism, Léon Poliakov describes John Chrysostom's Discourses against Judaizing Christians as « invectives of unbelievable violence » showing the «theological antisemitism » of the Fathers of the Church. However, Poliakov seems to ignore the literary traditions which influenced Chrysostom's Discourses. So, while they paid a tribute to the anti-Judaic polemical tradition which had characterized Christian literature since its beginning, the Discourses also depended on the pagan rhetorical tradition, which was conveyed in the teaching received by 4 th-century Christian writers. This literary tradition, emphasizing the importance of clarity and reasonable use of passions, was to Chrysostom a powerful way to promote his point of view in the turbulent and violent religious context of Antioch. Thanks to the reconsideration of both of these traditions, we can bring to light what the real meaning of Chrysostom's discourses is: they were an Antiochean priest's contribution to the construction of a Christian identity. Therefore, if we study the context of the Discourses' elaboration and place them in the uninterrupted chain of the tradition, we can demonstrate these texts do not attest to « theological antisemitism », as Poliakov said, but are an intensive and vivid reflexion about Christian identity in Late Antiquity.
KeYWORDS

John Chrysostom, anti-Judaic polemics, ancient rhetoric, Christianism, Late Antiquity. 
Les huit sermons de Jean Chrysostome regroupés par les éditeurs sous le titre Adversus Iudaeos sermones [1] jouissent encore de nos jours d'une réputation sulfureuse : nombreux sont ceux qui y voient ainsi les prodromes de l'antisémitisme le plus brutal. Parmi eux, Léon Poliakov reste le plus illustre et le plus influent. Dans son Histoire de I'antisémitisme, il qualifie ainsi les Homélies contre les juifs et les judaïsants de « diatribes d'une violence inimaginable »[2] et va jusqu'à qualifier I'antijudaïsme des Pères de l'Église $\mathrm{d}^{\prime}$ « antisémitisme théologique » [3]. Néanmoins, interpréter ainsi ces textes sous-estime la dimension topique de l'antijudaïsme antique et les isole de leur contexte, pourtant fondamental pour leur juste compréhension. Nous nous proposons donc de réfuter ici cette interprétation sommaire des Discours contre les juifs et les judaïsants et de démontrer que, loin d'être l'expression personnelle d'une rancœur tenace, ces textes sont en fait le réceptacle de traditions multiples et complémentaires qui dépassent largement leur auteur [4]. Ainsi, s'il est évident que ces textes entretiennent des rapports étroits avec la polémique antijuive, on ne peut faire l'économie de l'étude de la tradition rhétorique dans I'élaboration des Discours [5]. Étudier ces deux traditions nous permettra de voir enfin comment la rhétorique et la polémique sont mises au service d'une tentative de définition de l'identité chrétienne indissociable de la situation de la communauté chrétienne d'Antioche à la fin du IVe siècle.

[1] Ces discours datent des débuts de la carrière de Jean Chrysostome (386/387 apr. J.-C.). Pour une datation plus précise des huit sermons, voir PRADELS, BRÄNDLE \& HeImgartner 2002. Il est largement établi aujourd'hui qu'il faut exclure le troisième sermon comme relevant d'autres thématiques. La traduction des textes cités dans cet article, sauf mention contraire, est personnelle.

[2] Poliakov 1981, volume I, p. 33

[3] Ibid., p. 32. Les analyses de L. Poliakov ont ainsi influencé de nombreux savants qui ont entrepris une histoire globale de l'antisémitisme et ont cherché à en analyser les liens avec l'antijudaïsme chrétien antique et médiéval. Cependant, cette perspective diachronique manque radicalement la spécificité de la période antique. Parmi les études qui

\section{LES DISCOURS, HÉRITIERS DE LA TRADITION POLÉMIQUE ANTIJUDAÏQUE}

La postérité a surtout retenu des Discours le fait qu'ils prenaient les juifs pour cible. Il nous faut donc tenter dans un premier temps de situer ces textes dans cette tradition de la polémique antijuive [6]. Au IV siècle, cette catégorie littéraire possède depuis longtemps de nombreux représentants : du Dialogue avec Tryphon de Justin [7] aux Testimonia adversus Iudaeos du pseudo-Grégoire de Nysse [8], elle a pris des figures variées. Néanmoins, I'objectif a toujours été le même et il a été double : défendre la lecture chrétienne des Écritures et définir la spécificité du christianisme par rapport au judaïsme. Ce double objectif a donc conduit les auteurs chrétiens à développer un arsenal argumentatif permettant de légitimer et de fortifier leurs positions théologiques et exégétiques. S'est ainsi constituée au fil du temps une série de dossiers thématiques s'appuyant sur des citations des deux Testaments : la critique du ritualisme juif, la vocation des nations ou encore la naissance virginale du Christ ont ainsi fait l'objet de recueils de testimonia scripturaires dont chaque auteur pouvait se servir à sa guise et selon les circonstances [9]. Jean Chrysostome n'a pas dérogé à la règle.

tiennent de ce courant historiographique, nous pouvons citer BRUstein 2003 ou Laqueur 2006.

[4] Nous nous placerons ici dans le sillage des nombreux travaux qui ont souligné la singularité de l'antijudaïsme patristique et tenté d'en analyser les mécanismes rhétoriques et exégétiques : on peut citer MORLET 2014 ou, sur les Discours contre les juifs, BADY 2017.

[5] WILKEN 1983 ; MAXWELL 2006.

[6] Sur la littérature polémique antijuive, voir SCHRECKENBERG 1999 et LUKYN WiLLIAMS 1935.

[7] Sur ce texte, voir en particulier MunNICH 2013.

[8] Reynard 2015.

[9] Sur la question du recueil de testimonia comme genre littéraire, voir ALBL 1999. 
Pour illustrer ce point, un seul exemple suffira : celui d'une citation du livre de Malachie (MI 1, 10-11), habituellement citée comme une prophétie de la fin du culte sacrificiel et de la destruction du Temple de Jérusalem [10]:

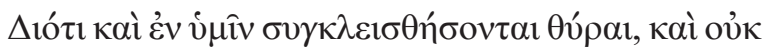

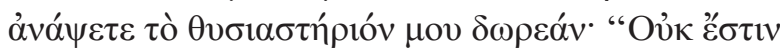

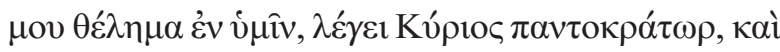

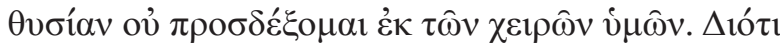

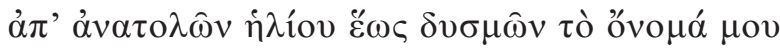

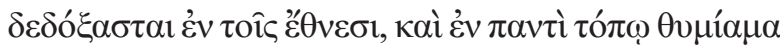

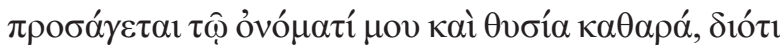

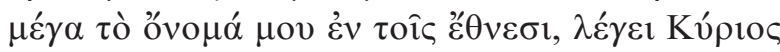
$\pi \alpha \nu \tau о \kappa \rho \alpha ́ \tau \omega \rho$.

De cette citation, nous allons étudier trois occurrences. D'abord, Jean Chrysostome I'invoque dans le quatrième Discours [11] :

"E

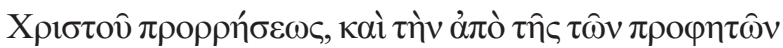

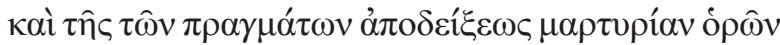

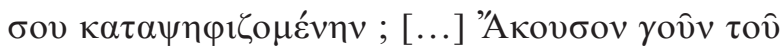

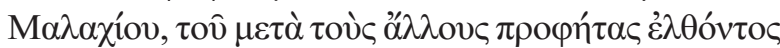

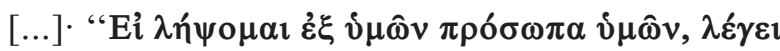

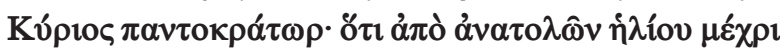

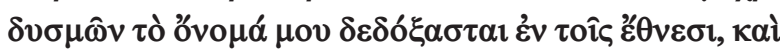

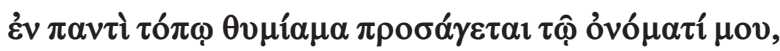

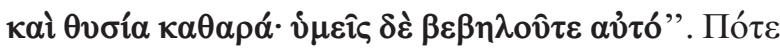

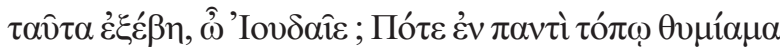

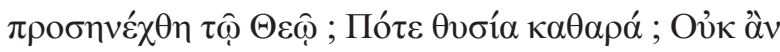

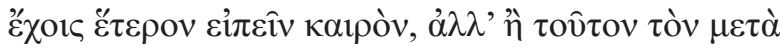

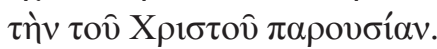

On pourrait simplement lire ce passage comme une violente prise à partie d'un interlocuteur juif : la citation de Malachie viendrait ainsi légitimer cette attaque et lui donner un surcroît d'autorité. Néanmoins, cet appel aux prophètes vétérotestamentaires fonde l'exercice même de la polémique avec les juifs et permet toujours d'étayer un argument traditionnellement invoqué par les chrétiens : I'aveuglement des juifs devant ce qu'ils considèrent comme de la clairvoyance prophétique.

En outre, il est possible de retrouver cette citation au début du IVe siècle, dans la Démonstration évangélique d'Eusèbe de Césarée, vaste ouvrage apologétique « structuré autour des grands dossiers de la polémique antijuive »[12]. Dans le livre I, il évoque la destruction du Temple de Jérusalem par les Romains et y voit une confirmation des prophéties. Après avoir cité une parole du Christ dans l'évangile de Jean (Jn 4, 23-24), Eusèbe poursuit [13] :

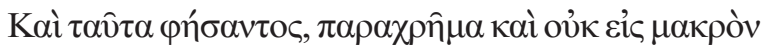

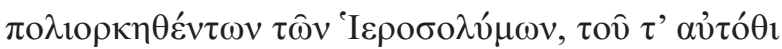

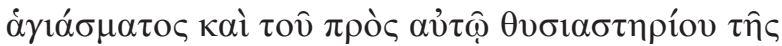

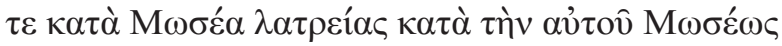

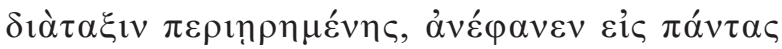

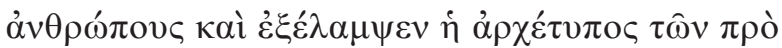

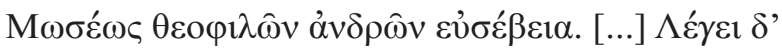
ơิv ơv

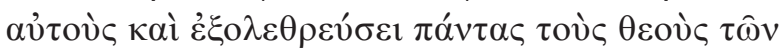

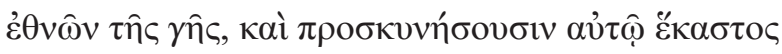

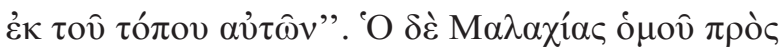

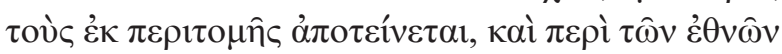

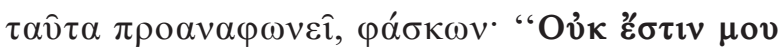

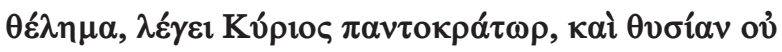

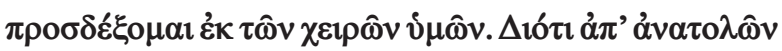

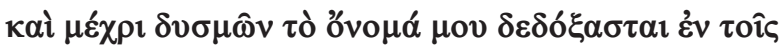

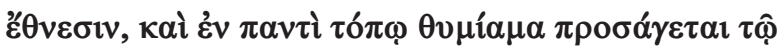

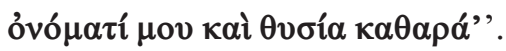

[10] RAHLfs \& HANHART, 2006, volume II, p. 562 : « C'est pourquoi on fermera sur vous les portes et vous n'allumerez pas en vain le feu de mon autel. "Je ne me complais pas en vous, dit le Seigneur tout-puissant, et je n'accueillerai pas le sacrifice de vos mains. C'est pourquoi depuis le Levant jusqu'au Couchant, mon nom a été glorifié parmi les nations ; en tout lieu on brûle de l'encens en mon nom, on m'apporte des victimes pures, parce que mon nom est grand parmi les nations", dit le Seigneur tout-puissant ». [11] Jean Chrysostome, Discours contre les juifs et les judaïsants, V, 12 (Patrologia Graeca, vol. 48, col. 901, I. 58-col. 902, I. 30) : « Et tu discutes encore, juif, alors que tu vois bien que le témoignage de la prophétie du Christ, que celle de la démonstration tirée des prophètes et des faits plaident contre toi ? [...] Écoute donc Malachie, qui est venu après les autres prophètes [...] : "]e ne recevrai plus des victimes de votre part, dit le Seigneur tout-puissant depuis le Levant jusqu'au Couchant, mon nom a été glorifié parmi les nations; en tout lieu on brûle de l'encens en mon nom, on m'apporte des victimes pures ; mais vous, vous avez profané [mon temple]". Quand cela s'est-il réalisé, juif ? Quand l'encens a-t-il été offert à Dieu en tout lieu ?
Quand lui a-t-on apporté des victimes pures ? Tu ne pourrais dire en d'autres temps que ceux qui ont suivi la venue du Christ 》.

[12] MORLET 2009, p. 37.

[13] Eusèbe de Césarée, Démonstration évangélique, I, 6, 41-43 (Heikel 1913, p. 29) : «Il dit cela, et aussitôt, peu de temps après, on faisait le siège de Jérusalem, on y détruisait le lieu saint, le sanctuaire qui s'y trouvait, sanctuaire associé au culte commandé par Moïse, placé là selon les ordres du même Moïse ; apparut alors de manière éclatante à tous les hommes le modèle de la dévotion des hommes pieux qui ont vécu avant Moïse. [...] Sophonie le dit clairement : "Le Seigneur leur apparaîtra et détruira tous les dieux des nations de la terre, et chacun se prosternera devant lui de son lieu". De la même manière, Malachie s'adresse à ceux de la circoncision [i.e. les juifs] et, à propos des nations, leur prophétise ceci : "Il n'y a, dit-il, aucun plaisir en moi, dit le Seigneur Tout-Puissant, et je n'accueillerai pas le sacrifice de vos mains. C'est pourquoi depuis le Levant jusqu'au Couchant, mon nom a été glorifié parmi les nations ; en tout lieu on brûle de l'encens en mon nom, on m'apporte des victimes pures" ». 
Ici encore, la citation de Malachie vient confirmer I'analyse du polémiste et se trouve même renforcée par une autre citation vétérotestamentaire, tirée du livre de Sophonie (So 2, 11) qui vient à la fois confirmer et compléter le message que porte Eusèbe : les prophètes ont annoncé la fin du culte sacrificiel et donc, selon lui, la fin du judaïsme. Invoquer plusieurs citations à la fois revient ainsi pour les chrétiens à souligner à la fois la cohérence de I'Ancien Testament et la justesse de leur interprétation des prophéties.

Enfin, cette idée apparaît chez Augustin, dans son Tractatus adversus Iudaeos. En effet, comme Eusèbe et Jean, le Père africain lit cette citation comme une annonce du culte tout spirituel lié au christianisme. Ce qui semble être une évidence pour lui le pousse à s'adresser vivement aux juifs [14] :

Postremo si haec verba prophetica secundum cor vestrum in alium sensum detorquere conamini, o Iudaei, contra salutem vestram resistentes Filio Dei ; [...] si haec ergo sic intelligere vultis, quid dicturi estis, et quomodo alium prophetam intellecturi, qui vobis omnino hanc amputat vocem, tanta manifestatione clamantem : "Non est mihi voluntas in vobis, dicit Dominus omnipotens, et sacrificium non accipiam de manibus vestris. Quia ab oriente sole usque in occidentem nomen meum clarum factum est in Gentibus; et in omni loco sacrificium offertur nomini meo, sacrificium mundum: quoniam magnum nomen meum in Gentibus, dicit Dominus omnipotens" ? Qua

tandem voce tantae rerum evidentiae reclamatis? Dans ce texte (qui se présente pourtant comme un traité, et non un discours), Augustin cherche à donner une présence aux adversaires en les apostrophant : il confirme ainsi que le propos leur est bien destiné [15]. Cette interpellation directe de l'adversaire n'est pas sans rappeler les Discours de Jean Chrysostome qui s'adressaient, quant à eux, directement à un auditoire. En outre, les termes ici employés par Augustin

[14] Augustin d'Hippone, Tractatus adversus Iudaeos, 9, 12 (Patrologia Latina, vol. 42, col. 60, I. 51-col. 61, I. 18 ; I'ensemble du texte est reproduit aux colonnes 51-64 de ce volume) : «Enfin, juifs, si vous cherchez à détourner ces paroles prophétiques selon votre cœur, dans un sens qui n'est pas le leur, résistant ainsi au Fils de Dieu contre l'intérêt de votre salut ; [...] si donc vous voulez comprendre ces paroles, que direz-vous, comment comprendrez-vous cet autre prophète qui vous laisse totalement sans voix en criant avec une si grande clarté : "Je n'ai pas d'affection pour vous, dit le Seigneur toutpuissant, et je ne recevrai pas de sacrifices de vos mains : car, depuis le Levant jusqu'au Couchant, mon nom est devenu éclatant parmi les nations et en tout lieu l'on offre un sacrifice en mon nom, un sacrifice pur, parce que mon nom est grand parmi les nations, dit le Seigneur tout-puissant." ? Quelle parole opposerez-vous à des faits si évidents ? ». renvoient aux mêmes lieux communs (I'obstination, l'aveuglement, la résistance à l'appel venu de Dieu), ce qui montre bien toute la dimension topique des attaques chrétiennes contre les juifs.

Étudier trois des occurrences de MI 1, 10-11 dans la littérature patristique nous permet ainsi d'ores et déjà d'apercevoir combien les thématiques et les arguments invoqués dans les Discours contre les juifs et les judaïsants relèvent d'un discours conventionnel que Jean Chrysostome reprend à son compte. Cependant, le rapprochement que nous venons de faire avec le traité d'Augustin nous permet d'aller plus loin et d'aborder un autre point : I'influence de la tradition rhétorique dans les Discours contre les juifs et les judaïsants.

\section{LES DISCOUUR, DES TEXTES MARQUÉS PAR L'HÉRITAGE RHÉTORIQUE CLASSIQUE}

Sur ce point, nous pouvons d'abord rappeler qu'on a rapidement fait de Jean Chrysostome l'un des disciples de Libanios, généralement considéré comme le dernier grand rhéteur de langue grecque de l'Antiquité [16] : cette tradition l'inscrit ainsi clairement dans le prolongement et la continuité de la tradition rhétorique. Cette dernière se caractérise par une élaboration progressive de techniques d'enseignement de l'art oratoire qui aboutit à la définition de genres rhétoriques variés. Dans cette perspective, les Discours contre les juifs et les judaïsants ont pour objectif principal de détourner du judaïsme les fidèles qui pourraient être séduits par ce culte en leur faisant une description peu flatteuse des juifs : il faut donc naturellement se tourner vers le genre rhétorique du blâme. Comme ce genre «se tire des prémisses opposées à celles de l'éloge » [17], nous pouvons l'envisager comme un négatif (au sens photographique) de l'éloge, à la fois proche et totalement opposé. Aristote écrit par exemple dans le livre I de la Rhétorique [18]:

[15] Sur la position d'Augustin envers les juifs et le judaïsme, voir BLUMENKRANZ 1958.

[16] Sur la figure essentielle de Libanios et sur son œuvre, voir PeTIT 1955 et 1956. On peut aussi renvoyer le lecteur à Festugiere 1959, qui envisage à la fois Libanios et Chrysostome.

[17] Aristote, Rhétorique, I, 1368a35 (dir. PelLegrin 2014, p. 2634). Nous renvoyons aussi le lecteur à PERNOt 1993, p. $481-490$.

[18] Aristote, Rhétorique, I, 1367a20 (dir. PelLegrin 2014, p. 2632) : «Puisque l'éloge se tire des actions et que le propre de l'homme de bien est d'agir par choix délibéré, il faut essayer de montrer que la personne qu'on loue agit par choix délibéré. Il est utile aussi de faire apparaître qu'elle a souvent agi de la sorte. Aussi faut-il traiter les coïncidences et les hasards comme des actes délibérés ». 


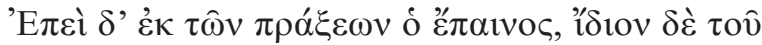

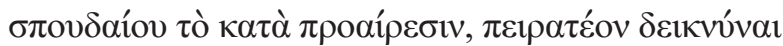

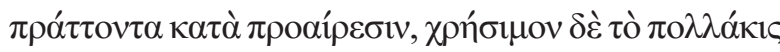

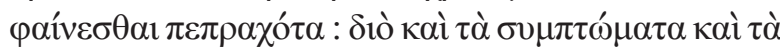

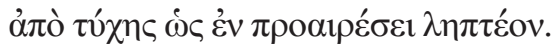

Nous voyons ici défini l'un des nombreux moyens de l'éloge : la flatterie. Si la vérité n'est pas à l'avantage du destinataire, il est du devoir de l'orateur de le présenter sous son plus beau jour, en dépit même de la vérité. Néanmoins, cette flatterie a son revers, son négatif : le dénigrement. S'il veut faire une présentation négative de son adversaire, le rhéteur doit forcer le trait et parfois s'éloigner de la vérité. Il atteint ainsi son objectif : persuader ses auditeurs et les faire adhérer à sa position. On peut donc dire que le mensonge était érigé au rang de technique oratoire indispensable, autant dans l'éloge que dans le blâme.

À la même époque que Jean Chrysostome, Libanios nous en donne un exemple éclatant dans son discours Pro templis [19] :

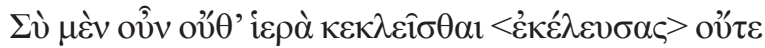

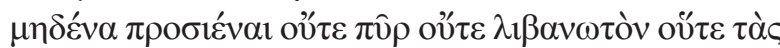

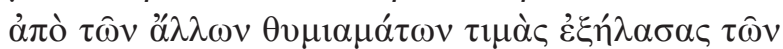

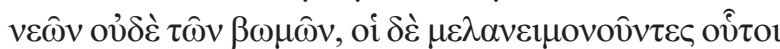

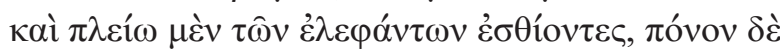

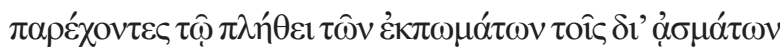

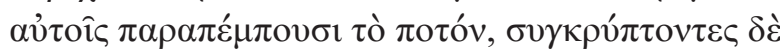

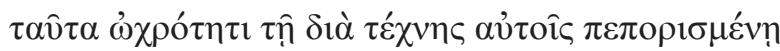

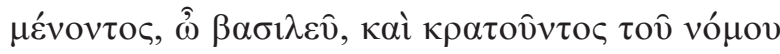

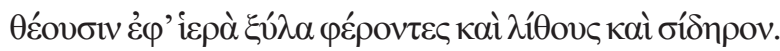
Dans le cadre d'une querelle autour de la fermeture des temples païens, Libanios, rhéteur païen, présente ici les moines chrétiens comme des hommes rustres, violents et gloutons : ils font ici office de figures-repoussoirs censées symboliser ce que le christianisme peut produire de plus opposé à la civilité grecque telle que l'idéalise Libanios. Ce dernier les caricature en les rendant parfaitement antipathiques et s'attire ainsi la sympathie de son auditoire. Mais qu'en est-il chez Jean Chrysostome ? Qu'en est-il dans les Discours contre les juifs et les judaïsants ?

On trouve chez ce dernier des descriptions qui, sorties de leur contexte, se distinguent par leur très grande violence. L'exemple le plus fréquemment cité est tiré du premier Discours [20] :

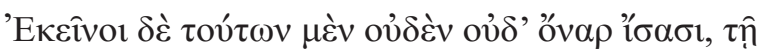

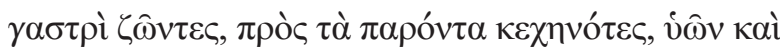

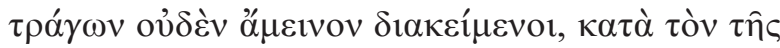

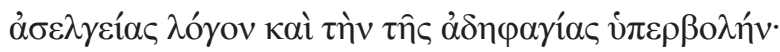

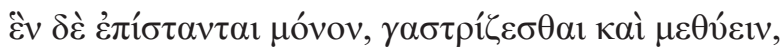

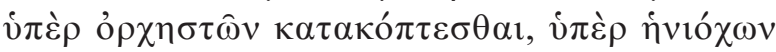
$\tau \rho \alpha v \mu \alpha \tau i \zeta \varepsilon \sigma \theta \alpha$.

Le thème de l'obstination des juifs à ne pas reconnaître la vérité du christianisme s'assortit ici d'une attaque en règle de leurs mœurs supposées : avidité, intempérance, ivresse sont autant de thèmes qui renvoient les adversaires de l'orateur aux limites de la civilisation et de l'humanité (tout comme les moines chrétiens chez Libanios). Jean Chrysostome ne réserve cependant pas ses traits aux juifs. En témoigne un autre discours, le Discours sur Babylas, qui présente les païens sous un jour peu flatteur [21] :

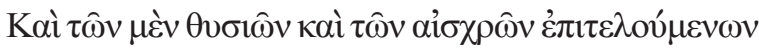

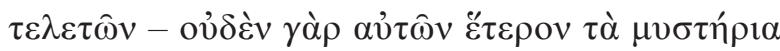

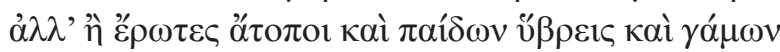

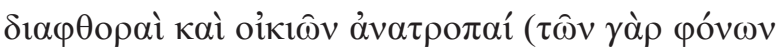

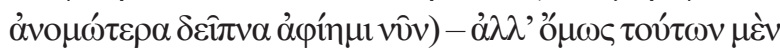

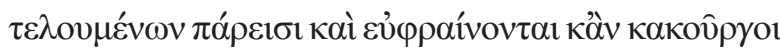

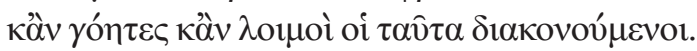

Confronter ces deux passages revient à faire un constat : les propos de Jean Chrysostome, s'ils paraissent excessifs et brutaux, ne tiennent pas tant de l'insulte que de la stratégie de persuasion. Le dénigrement permet ici à l'orateur de discréditer son adversaire auprès de ses auditeurs et d'acquérir ainsi en retour un surcroît d'autorité.
[19] Libanios, Orationes, XXX, 8 (nous citons le texte grec édité dans Foerster 1906, p. 91) : « Toi donc tu n'as ni fait fermer les temples ni interdit leur accès ; tu n'as banni des temples et des autels ni le feu, ni l'encens, ni les autres offrandes de parfums ; et cependant ces hommes vêtus de noir qui mangent plus que des éléphants et qui fatiguent, par la quantité de coupes qu'ils vident, ceux qui leur servent à boire au milieu des chants et qui cachent leurs désordres sous une pâleur artificielle, ces gens-là, ô empereur, au mépris de la loi qui reste toujours en vigueur courent vers les temples, portant des morceaux de bois, des pierres et du fer » (traduction française publiée dans VAN LOY 1933, p. 22).

[20] Jean Chrysostome, Discours contre les juifs et les judaïsants, I, 4 (Patrologia Graeca, vol. 48, col. 848, I. 50-col. 849, I. 4) : « Ces gens-là [i.e. les juifs] ne savent rien de ces choses, pas même l'ombre ; ils vivent pour leur ventre, ils sont affamés des biens présents, ils ne se comportent pas mieux que des porcs et des boucs et suivent la voix de leurs mœurs dissolues et l'excès de leur voracité. Ils ne savent qu'une chose : s'empiffrer et s'enivrer, se battre pour des danseurs, se blesser pour des cochers ».

[21] Jean Chrysostome, Sur Babylas, 74 (SCHATKIN, BLANC \& GRILLET 1990, p. 190-193) : «Quand sont célébrés les sacrifices et les cérémonies honteuses - leurs mystères ne sont rien d'autres qu'amours anormales, outrages à de jeunes garçons, ruine des mariages et bouleversement des foyers! car je laisse de côté, pour l'instant, les rites sinistres de leurs meurtres et leurs repas plus criminels que les meurtres -, quand on les célèbre, cependant, [les démons] sont là, tout heureux, quand bien même ceux qui les célèbrent sont des malfaiteurs, des charlatans, de vrais fléaux ». 
Mais pour y parvenir, Jean Chrysostome use aussi de l'exemple édifiant, de l'anecdote significative. De nouveau, le premier Discours nous en donne une illustration [22] :

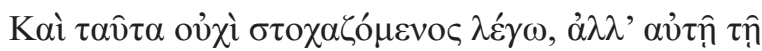

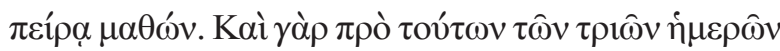

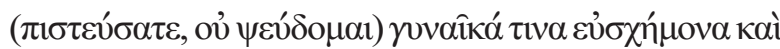

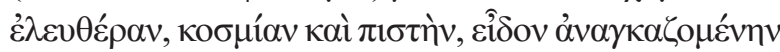

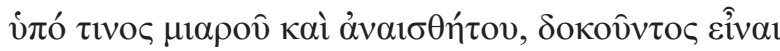

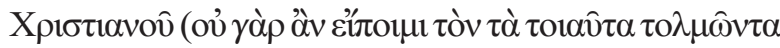

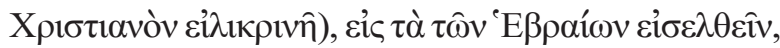

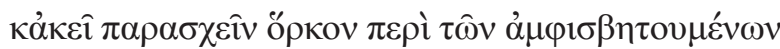

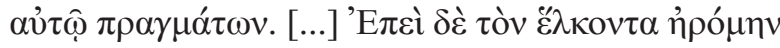

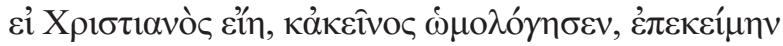

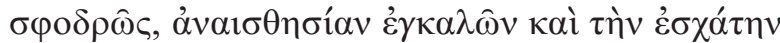

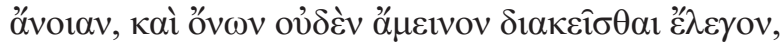

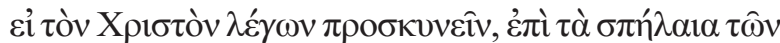

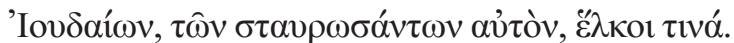

Ce passage est intéressant à de multiples titres. D'abord, il donne au message pastoral de Jean Chrysostome une illustration extrêmement concrète, puisqu'elle fait appel à des personnages, à des lieux et à des actions bien connus des fidèles et qu'elle est censée s'appuyer sur l'expérience. Il met ensuite en scène les luttes que doivent mener les chrétiens contre ceux de leurs coreligionnaires qui veulent les attirer à la synagogue. Enfin, il donne à l'orateur un rôle actif, puisque c'est lui qui prend la défense de la victime et confond le coupable. Cette anecdote, tout comme le passage précédent du premier Discours, confirme donc bien l'enracinement des Discours dans la tradition rhétorique et le triple objectif que se fixe Jean Chrysostome : plaire à I'auditoire en s'attirant sa bienveillance ; I'émouvoir en I'incitant à prendre son parti ; enseigner les vérités de la foi chrétienne et la pratique religieuse qui en découle.

\section{LES DISCOURS, UN INSTRUMENT DE DÉFINITION IDENTITAIRE}

Perdre de vue la dimension à la fois didactique et parénétique des Discours contre les juifs et les judaïsants pour les réduire à un réquisitoire incendiaire revient en effet à ignorer complètement le contexte qui les a vu naître, et donc leur raison d'être même. Pour reprendre les mots d'Emmanuel Soler, qui a consacré un ouvrage majeur à la vie religieuse à Antioche, « I'objet premier des homélies Contre les Juifs est d'ordre théologique »[23] : il nous faut donc nous mettre dans cette perspective pour lire ces textes. De plus, outre les juifs, sont évoqués dans les Discours une autre catégorie religieuse : celle des judaïsants.

Antioche était à la fin du IVe siècle une métropole prospère. Cette prospérité allait de pair avec une forte concentration de population, et donc une bonne représentation des trois religions principales de l'Empire : le paganisme, le christianisme et le judaïsme [24]. Cette dernière était particulièrement présente, du fait d'une installation de longue date des juifs dans cette métropole [25], et le judaïsme antiochien se distinguait par la place importante qu'il consacrait à la religiosité de rue, particulièrement à l'occasion des grandes fêtes. Cette visibilité accrue a conduit certains chrétiens à se rapprocher des communautés juives, allant jusqu'à prendre part à leurs fêtes et à aller à la synagogue tout autant qu'à l'église [26] : il s'est donc constitué au sein de la communauté chrétienne d'Antioche un groupe de chrétiens judaïsants ou « demi-chrétiens » [27]. Jean Chrysostome nous décrit cette habitude sous un jour négatif dans le quatrième Discours [28] :
[22] Jean Chrysostome, Discours contre les juifs et les judaïsants, I, 3 (Patrologia Graeca, vol. 48, col. 847, I. 39-60) : « Et je ne dis pas cela sur une simple conjecture, mais je l'ai appris de l'expérience même. Ainsi, il y a trois jours (croyezmoi, je ne mens pas), j'ai vu une femme honnête et libre, digne et pieuse contrainte par un homme infâme et stupide, qui semblait être chrétien (je ne m'aventurerais pas en effet à dire qu'un chrétien sincère oserait agir de la sorte) à aller à la synagogue des Hébreux et y recevoir d'elle un serment à propos d'une affaire incertaine pour lui. [...] Lorsque je demandai à l'agresseur s'il était chrétien, et que lui reconnut l'être, je le pressai avec force en lui reprochant sa stupidité et sa profonde folie, et je lui disais qu'il ne se comportait pas mieux que les ânes si, alors qu'il disait adorer le Christ, il entraînait quelqu'un dans les antres des juifs qui I'ont crucifié ». [23] SOLER 2006, p. 107.

[24] Sur ce point, nous pouvons citer Meeks \& WiLken 1978 et VAN DER HORST 2000.

[25] On peut constater cette présence dès la fondation de la cité, comme nous l'apprend Flavius Josèphe (Flavius Josèphe, Antiquités juives, XII, 3, 1).
[26] Il y a de nombreuses attestations de ce phénomène : les plus significatives pour notre propos sont celles qui sont évoquées dans les Constitutions apostoliques, dont la compilation semble avoir été faite à Antioche au début du IVe siècle (voir par exemple Constitutions apostoliques, 2, 61, 1 ou 8, 47, 65). [27] Nous empruntons cette expression à Laurence Brottier, qui I'utilise à plusieurs reprises : BROTTIER 2004 et 2005.

[28] Jean Chrysostome, Discours contre les juifs et les judaïsants, IV, 7 (Patrologia Graeca, vol. 48, col. 881, I. 36-col. 882 , I. 4) : «Que cours-tu donc voir à la synagogue des juifs, des ennemis de Dieu, dis-moi ? Des hommes soufflant dans des trompettes ? Alors que vous devriez rester chez vous, gémir et pleurer sur ces gens, parce qu'ils combattent l'injonction de Dieu, parce qu'ils ont le diable qui danse avec eux. [...] Où est donc l'autel ? Où est I'arche ? Où sont le tabernacle et le Saint des saints ? Où est le prêtre ? Où sont les chérubins de gloire ? Où est l'autel des parfums, couvert d'or ? Où est le propitiatoire ? Où est la coupe ? Où sont les vases à libations ? Où est le feu venu d'en haut ? Tu as laissé disparaître tout cela, mais tu gardes seulement les trompettes ? Vois-tu que c'est davantage de l'enfantillage qu'un culte ? ». 


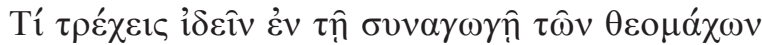

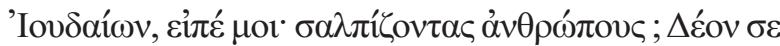

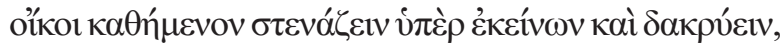

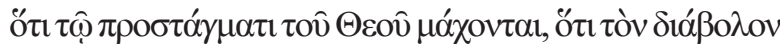

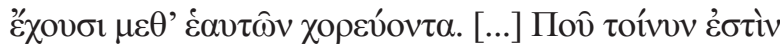

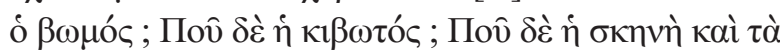

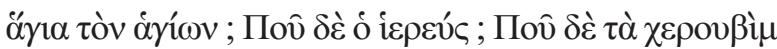

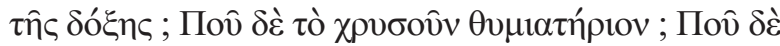

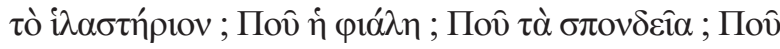

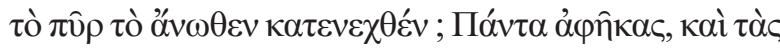

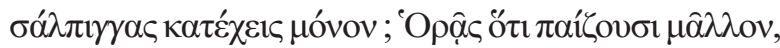
ì $\lambda \alpha \tau \rho \varepsilon v ́ o v \sigma o v$;

Il s'agit bien de faire cesser tout contact festif des fidèles avec les autres communautés. Le prêtre mène donc une « guerre de concurrence avec les juifs » [29], dans laquelle tous les coups sont permis. Face à un auditoire sans cesse mouvant et toujours composite [30], il est difficile pour un prêtre comme Jean Chrysostome de définir I'identité chrétienne à partir de postulats théologiques partagés par tous. Dans ces conditions, comment définir cette identité ? Pour Jean Chrysostome, la définition de cette identité passe essentiellement par l'usage de la parole, et de la parole sous sa forme la plus élaborée : la rhétorique [31]. Dans son traité Sur le sacerdoce, Jean Chrysostome lui-même théorise cet usage en l'assimilant de manière suggestive à l'activité guerrière [32]:

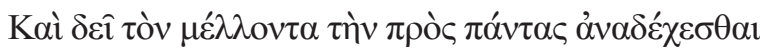

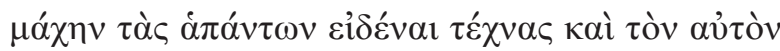

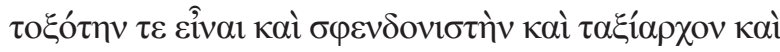
$\lambda$

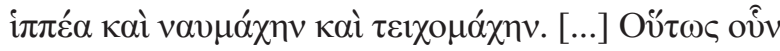

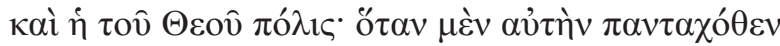

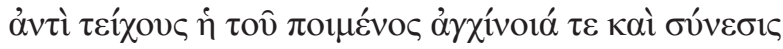

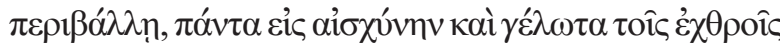

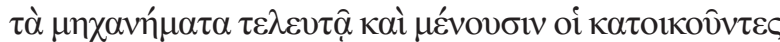

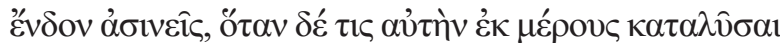

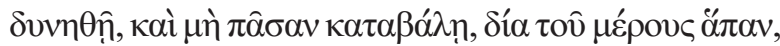

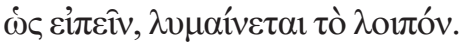

[29] BRÄNDLE 2018, p. 303.

[30] Sur ce point, nous renvoyons le lecteur à PERRIN 2017. Toutefois, Jean Chrysostome le constatait déjà lui-même dans son traité Sur le sacerdoce : le prédicateur doit en effet « lutter souvent contre l'inexpérience de tout un peuple. » (Sur le sacerdoce, V, 6 (MALINGRey 1980, p. 295-296)).

[31] Cet usage de la rhétorique peut aussi s'expliquer par une persistance de la civilité païenne à Antioche, marquée par la place du jeu et de la parole : nous renvoyons le lecteur à NATALI 1985.

[32] Jean Chrysostome, Sur le sacerdoce, IV, 4 (MALINGREY 1980 , p. 252-255) : «Celui qui doit soutenir le combat contre tous doit connaître toutes les tactiques ; il doit être à la fois archer et frondeur, commandant d'un corps d'armée et d'une petite unité, soldat et stratège, fantassin et cavalier, matelot et défenseur des remparts. [...]. Il en est ainsi de la cité de Dieu ; lorsque, au lieu des remparts, l'intelligence et la pénétration du berger l'environnent,
Ce texte souligne plusieurs éléments importants pour notre propos. La comparaison du ministère de la prédication avec la chose militaire et la poliorcétique renvoie ainsi clairement à l'activité polémique du prêtre. De la même manière, ce passage évoque de manière allusive le risque de laisser grandir au sein de l'Église des opinions hétérodoxes qui pourraient nuire à l'ensemble des fidèles et à l'unité de la communauté.

Il s'agira donc non pas de transmettre aux fidèles des enseignements théologiques abstraits, mais plutôt de leur inculquer une pratique religieuse. L'identité chrétienne se placera moins sur le plan théorique que sur celui de la praxis [33] : participer aux jeûnes des juifs, c'est judaïser, c'est devenir soi-même juif et s'éloigner ainsi de la foi chrétienne [34]. La polémique et la rhétorique deviennent donc pour Jean Chrysostome des instruments pour réagir à une conception selon lui trop souple de l'identité religieuse et pour présenter un modèle alternatif fondé principalement sur le contraste avec l'adversaire et sur l'orthopraxie.

\section{CONCLUSION}

L'histoire de la réception des Discours contre les juifs et les judaïsants de Jean Chrysostome est celle d'un malentendu [35]. La postérité, évacuant la question des judaïsants et de la définition de l'identité chrétienne comme trop circonstancielle, a préféré accorder son attention à la cible secondaire : les juifs. Jusqu'à une époque récente, on a pu ranger Jean Chrysostome dans la galerie des intolérants [36]. Cependant, c'est là se tromper. Les travaux les plus récents soulignent en effet très nettement la complexité des identités religieuses antiochiennes, souvent marquées par l'entre-deux [37]. Dans ce contexte, si la violence des propos de Jean Chrysostome laisse parfois penser qu'elle dépasse le cadre des lieux communs rhétoriques, les Discours contre les juifs et les judaïsants ne relèvent néanmoins

toutes les ruses des ennemis tournent à leur honte et à leur dérision et ceux qui l'habitent restent à l'intérieur à I'abri de tout dommage, mais lorsque quelqu'un peut la détruire en partie, même s'il ne la détruit pas tout entière, il suffit, pour ainsi dire, d'une partie pour que l'ensemble soit ruiné ».

[33] Isabella Sandwell décrit ce phénomène comme une « great emphasis on visible behaviour » (SANDWELL 2007, p. 73). Cette définition de l'identité chrétienne par la praxis s'explique d'autant mieux que distinguer orthodoxes et hétérodoxes au sein même des communautés chrétiennes n'était pas toujours facile (PERRIN 2017, p. 112-121).

[34] Jean Chrysostome, Discours contre les juifs et les judaïsants, I, 1 (Patrologia Graeca, vol. 48, col. 845).

[35] BRÄNDLE \& PRADELS, 2008.

[36] Athanassiadi 2010, p. 96.

[37] SANDWELL 2007, passim. Cette lecture de la vie religieuse antiochienne est aussi défendue dans Cote 2012. 
pas exclusivement de la subjectivité de leur auteur. Au contraire, ils reprennent des instruments à la tradition déjà largement affirmée pour les adapter au contexte particulier qui est le leur, celui de la cité d'Antioche. Si Jean Chrysostome mêle les traditions, on peut penser que les raisons en sont multiples. Néanmoins, elles peuvent toutes se rapporter à la christianisation progressive de la culture antique que vivent à la fois Jean et sa communauté : Jean, parce qu'il a été éduqué à la fois dans une famille chrétienne et dans un système éducatif encore marqué par la culture classique ; sa communauté, parce qu'elle conserve une vie sociale influencée par la civilité païenne et qu'elle possède une identité religieuse encore flottante. Plus largement, on peut dire que cette multiplicité des traditions qui irriguent les Discours contre les juifs et les judaïsants illustre la lente mutation de la culture chrétienne de la fin de I'Antiquité.

BIBLIOGRAPHIE

ALBL, Martin Christian, 1999, "And Scripture cannot be broken" : The Form and Function of the Early Christian Testimonia Collections, Leiden.

DOI : $10.1093 /$ jts/52.1.293

Athanassiadi, Polymnia, 2010, Vers la pensée unique. La montée de l'intolérance dans l'Antiquité tardive, Paris.

BADY, Guillaume, 2017, «Quelques éléments de réflexion sur les Sermons contre les juifs et les judaïsants de Jean Chrysostome » dans Jean-Marie Auwers, Régis Burnet \& Didier Luciani (éd.), L'antijudaïsme des Pères : mythe et/ou réalité ?, Paris, p. 101-118.

Blumenkranz, Bernhard, 1958, « Augustin et les juifs - Augustin et le judaïsme », Recherches Augustiniennes et Patristiques 1, p. 225-241.

BRÄNDLE, Rudolf, 2018, « Die Reden Adversus Iudaeos (386/387) von Johannes Chrysostomus im Kontext der multikulturellen Metropole Antiochien », dans Silke-Petra Bergjan \& Susanna Elm (éd.), Antioch II. The Many Faces of Antioch : Intellectual Exchange and Religious Diversity, CE 350-450, Tübingen, p. 297-316.

Brändle, Rudolf \& Pradels, Wendy, 2008, « Boshaft wie goldene Rede. Aspekte der Wirkungsgeschichte der Reden gegen die Juden von Johannes Chrysostomus », dans Martin Walraff \& Rudolf Brändle (éd.), Chrysostomosbilder in 1600 Jahren : Facetten der Wirkungsgeschichte eines Kirchenvaters, Berlin, p. 235-254.

BrotrIer, Laurence, 2004, « Jean Chrysostome : un pasteur face à des "demi-chrétiens" », Topoi. Orient-Occident, Supplément 5 , p. 439-457.

Brottrer, Laurence, 2005, L'appel des « demi-chrétiens » à la « vie angélique », Paris.

Brustern, William, 2003, Roots of Hate : Anti-Semitism in Europe before the Holocaust, Cambridge.

DOI : $10.1017 /$ cbo9780511499425.

Cote, Dominique, 2012, «Le problème de l'identité religieuse dans la Syrie du IVe siècle. Le cas des Pseudo-Clémentines et de I'Adversus Judaeos de saint Jean Chrysostome», dans Simon Claude Mimouni \& Bernard Pouderon (éd.), La croisée des chemins revisitée. Quand I'Église et la Synagogue se sont-elles distinguées ?, Paris, p. 339-370.

Festugrere, André-Jean, 1959, Antioche païenne et chrétienne : Libanios, Chrysostome et les moines de Syrie, Paris.

Foerster, Richard, 1906, Libanios. Opera. Vol. III. Orationes XXVI-L, Leipzig.

HeIKel, Ivar August, 1913, Eusebius Werke. Die Demonstratio evangelica, Leipzig.

LAqUEUR, Walter, 2006, The Changing Face of Antisemitism : From Ancient Times To The Present Day, Oxford.

Lukyn Wrlliams, Arthur, 1935, Adversus Iudaeos. A bird's-eye view of Christian Apologiae until the Renaissance, Cambridge. DOI : $10.1017 /$ cbo9781139108478.

Malingrey, Anne-Marie, 1980, Jean Chrysostome. Sur le sacerdoce, Paris.

Maxwell, Jaclyn LaRae, 2006, Christianization and Communication in Late Antiquity : John Chrysostom and his Congregation in Antioch, Cambridge.

DOI : $10.1017 /$ cbo9780511482854.

Meeks, Wayne Atherton \& WrLken, Robert Louis, 1978, Jews and Christians in Antioch in the first four centuries of the common era, Missoula (MT).

DOI : $\underline{10.2307 / 3164770 .}$ 
Morlet, Sébastien, 2009, La Démonstration évangélique d'Eusèbe de Césarée. Étude de l'apologétique chrétienne à l'époque de Constantin, Paris.

Morlet, Sébastien, 2014, «L'antijudaïsme chrétien au IVe siècle. À propos de quelques idées reçues », dans Marie-Françoise Baslez (éd.), Chrétiens persécuteurs. Destructions, exclusions, violences religieuses au IVe siècle, Paris, p. 163-188.

Munnich, Olivier, 2013, « Le judaïsme dans le Dialogue avec Tryphon : une fiction littéraire de Justin » dans Sébastien Morlet, Olivier Munnich \& Bernard Pouderon (éd.), Les dialogues Adversus Iudaeos. Permanences et mutations d'une tradition polémique, Paris, p. 95-158.

NatAlI, Alain, 1985, «Tradition ludique et sociabilité dans la pratique religieuse à Antioche d'après Jean Chrysostome », Studia Patristica 16, p. 463-470.

Patrologiae Cursus Completus. Series Latina, 1844-1855, Paris, 221 volumes.

Patrologiae Cursus Completus. Series Graeca, 1857-1866, Paris, 161 volumes.

Pellegrin, Pierre (éd.), 2014, Aristote. Fuvres complètes, Paris.

Pernot, Laurent, 1993, La rhétorique de l'éloge, Paris.

Perrin, Michel-Yves, 2017, Civitas confusionis. De la participation des fidèles aux controverses doctrinales dans l'Antiquité tardive, Paris.

Petrt, Paul, 1955, Libanius et la vie municipale à Antioche au IV siècle après J.-C., Paris.

DOI : $10.4000 /$ books.ifpo.5151.

Petrt, Paul, 1956, Les étudiants de Libanius. Un professeur de faculté et ses élèves au Bas Empire, Paris.

Poliakov, Léon, 1981, Histoire de l'antisémitisme, Paris.

Pradels, Wendy, Brändle, Rudolf \& Hetmgartner, Martin, 2002, « The Sequence and Dating of the Series of John Chrysostom's Eight Discourses Adversus Iudaeos », Zeitschrift für antikes Christentum 6, p. 90-116.

DOI : 10.1515/zach.2002.013.

RAHLFs, Albert \& HANHART, Robert, 2006, Septuaginta. Id est Vetus Testamentum juxta LXX interpretes, Stuttgart.

ReYnARD, Jean, 2015, «Le travail de l'extrait dans les Testimonia du pseudo-Grégoire de Nysse », dans Sébastien Morlet (éd.), Lire en extraits. Lecture et production des textes de I'Antiquité à la fin du Moyen Âge, Paris, p. 259-280.

SANDWell, Isabella, 2007, Religious identity in late Antiquity : Greeks, Jews and Christians in Antioch, Cambridge.

DOI : $10.1017 /$ cbo9780511482915.

Schatkin, Margaret Amy, Blanc, Cécile \& Grillet, Bernard, 1990, Jean Chrysostome. Sur Babylas, Paris.

Schreckenberg, Heinz, 1999, Die christlichen Adversus-Judaeos-Texte und ihr historisches und literarisches Umfeld (1.-11. Jhd), Frankfurt-am-Main.

Soler, Emmanuel, 2006, Le sacré et le salut à Antioche au IV siècle après J.-C. Pratiques festives et comportements religieux dans le processus de christianisation de la cité, Beirut.

VAN Der Horst, Pieter Willem, 2000, « Jews and Christians in Antioch at the End of the Fourth Century » dans Stanley E. Porter \& Brook W. R. Pearson (éd.), Christian-Jewish Relations through the Centuries, Sheffield, p. 228-238.

Van Loy, René, 1933, «Le "Pro templis" de Libanios », Byzantion 8/1, p. 7-39.

WrLKen, Robert Louis, 1983, John Chrysostom and the Jews. Rhetoric and reality in the late 4th century, Berkeley - Los Angeles. 